\title{
CRITÉRIOS DE BEERS-FICK E MEDICAMENTOS GENÉRICOS NO BRASIL
}

Milton Luiz Gorzoni*, Renato Moraes Alves fabbri, Suel Luciano Pires

Trabalho realizado no departamento de Clínica Médica, da disciplina de Gerontologia da Faculdade de Ciências Médicas da Santa Casa de São Paulo, São Paulo, SP

*Correspondência

R. Santo Irineu, 285,

apt 11 - Saúde

São Paulo - SP

CEP $04127-120$

Tel: (11) 5578-9328

Fax: (11) 3221-0325

gorzoni@uol.com.br

\section{RESUMO}

OBjEtIVo. Determinar a prevalência de fármacos potencialmente inapropriados para idosos em medicamentos genéricos brasileiros pelos critérios de Beers-Fick.

Métodos. Análise da lista de medicamentos genéricos publicada no Diário Oficial da União de 12 de julho de 2004 e copiada da página da Agência Nacional de Vigilância Sanitária (ANVISA) - www.anvisa.gov.br, utilizando-se os critérios de Beers-Fick. Resultados. Contendo 299 produtos e/ou apresentações, a lista analisada apresentava 20 deles (6,7\% do total) incluídos nos critérios de Beers-Fick, concentrados nas categorias de ansiolíticos, antiagregantes plaquetários, antialérgicos, antiangionosos e vasodilatadores, antiarrítmicos, antidepressivos, antiespasmódicos, anti-hipertensivos, antiinflamatórios não esteroidais, antiulcerosos e glicosídeos cardíacos. Esses critérios não incluem fármacos como antitussígenos, cinarizina, diltiazem, piracetam, quinolonas, xantinas, cremes, pomadas e colírios que fazem parte dessa lista de medicamentos genéricos.

Conclusäo: Critérios de Beers-Fick são úteis para a prevenção do uso de fármacos potencialmente inapropriados em idosos, com a ressalva de que não são completos para medicamentos genéricos brasileiros.

UnITERMOS: Idosos. Medicamentos. Prescrição. latrogenia.

\section{INTRODUÇão}

Pacientes idosos normalmente são acometidos por mais de uma doença simultaneamente e, devido a isso, tomam vários medicamentos de forma contínua e concomitantel, 1,3 . Multiplicidade de doenças e de fármacos associada a alterações relacionadas ao envelhecimento desencadeia, com constância, graves interações medicamentosas e reações adversas nesses pacientes ${ }^{4-7}$.

Estima-se que $5 \%$ do total de hospitalizações, independentemente da idade dos pacientes, decorram de reações adversas a medicamentos ${ }^{8,9}$. Observa-se, porém, que a probabilidade de internações hospitalares serem decorrentes de efeitos colaterais e/ ou interações medicamentosas dobra a triplica em idosos ${ }^{10,11,12}$. Relata-se também que entre metade e dois terços de idosos hospitalizados apresentarão pelo menos uma reação adversa a medicamentos $^{11,13}$.

Há aproximadamente duas décadas surgiram instrumentos visando detectar potenciais riscos de iatrogenia medicamentosa em idosos, sendo o de Beers-Fick o mais utilizado deles ${ }^{13-16}$. Beers et al. ${ }^{14,15}$ estabeleceram critérios, baseados em trabalhos publicados sobre medicamentos e farmacologia do envelhecimento, para definir lista de fármacos potencialmente inapropriados a adultos com 65 ou mais anos de idade. Fick et al. ${ }^{16}$ atualizaram esses critérios, dividindo-os em dois: I) Medicamentos ou classes deles que deveriam ser evitados em idosos, independentemente do diagnóstico ou da condição clínica, devido ao alto risco de efeitos colaterais e pela existência de outros fármacos mais seguros; 2) Medicamentos ou classes deles que não devem ser usados em determinadas circunstâncias clínicas. Pergunta-se se os critérios de Beers-Fick poderiam ser utilizados como guia para a boa prescrição de medicamentos genéricos brasileiros em idosos, procurando-se assim reduzir iatrogenias, consultas e hospitalizações nesses pacientes.

\section{Овjetivo}

Definir a aplicabilidade dos critérios de Beers-Fick para determinar a prevalência de fármacos potencialmente inapropriados para idosos em medicamentos genéricos brasileiros.

\section{Métodos}

Análise de lista de medicamentos genéricos publicada no Diário Oficial da União de 12 de julho de 2004 e copiada da página da Agência Nacional de Vigilância Sanitária (ANVISA) - www.anvisa.gov.br, utilizando-se os critérios de Beers-Fick ${ }^{16}$ para fármacos não recomendados em idosos, independentemente do diagnóstico ou da condição clínica, devido ao alto risco de efeitos colaterais e com opções à prescrição de outros fármacos mais seguros e comercializados no Brasil (Tabela I).

\section{Resultados}

Contendo 299 produtos e/ou apresentações, a lista analisada apresentava 20 deles (6,7\% do total) incluídos nos critérios de BeersFick, concentrados nas categorias de ansiolíticos, antiagregantes 


\begin{tabular}{|c|c|}
\hline \multicolumn{2}{|c|}{$\begin{array}{l}\text { Tabela I - Medicamentos não recomendados em idosos, } \\
\text { independentemente do diagnóstico ou da condição clínica, } \\
\text { devido ao alto risco de efeitos colaterais e com opções à } \\
\text { prescrição de outros fármacos mais seguros pelos } \\
\text { critérios de Beers-Fick }{ }^{14} \text { e comercializados no Brasil }\end{array}$} \\
\hline Benzodiazepínicos & Amiodarona \\
\hline Lorazepam > 3,0 mg/dia & Digoxina $>0,125 \mathrm{mg} / \mathrm{dia}$ \\
\hline Alprazolam > 2,0 mg/dia & (exceto em arritmias atriais) \\
\hline Clordiazepóxido & Disopiramida \\
\hline Diazepam & Metildopa \\
\hline Clorazepato & Clonidina \\
\hline Flurazepam & Nifedipina \\
\hline Amitriptilina & Doxazosina \\
\hline Fluoxetina(diariamente) & Dipiridamol \\
\hline Barbitúricos (excetofenobarbital) & Ticlopidina \\
\hline Tioridazina & Antïnflamatórios não-hormonais \\
\hline Meperidina & Indometacina \\
\hline Anoréxicos & Naproxeno \\
\hline Anfetaminas & Piroxicam \\
\hline Anti-histamínicos & Miorrelaxantes e antiespasmódicos \\
\hline Clorfeniramina & Carisoprodol \\
\hline Difenidramina & Clorzoxazona \\
\hline Hidroxizina & Ciclobenzaprina \\
\hline Ciproeptadina & Orfenadrina \\
\hline Tripelenamina & Oxibutinina \\
\hline Dexclorfeniramina & Hiosciamina \\
\hline Prometazina & Propantelina \\
\hline Clorpropamida & Alcalóides da Belladonna \\
\hline Estrogênios não-associados (via oral) & Cetorolaco \\
\hline Extrato de Tireóide & Ergote ciclandelata \\
\hline Metiltestosterona & Laxantes \\
\hline Nitrofurantoina & Bisacodil \\
\hline Sulfato ferroso & Cascará sagrada \\
\hline Cimetidina & Óleomineral \\
\hline
\end{tabular}

plaquetários, antialérgicos, antianginosos e vasodilatadores, antiarrítmicos, antidepressivos, antiespasmódicos, anti-hipertensivos, antiinflamatórios não esteroidais, antiulcerosos e glicosídeos cardíacos (Tabela 2). Esses critérios não incluem fármacos como antitussígenos, cinarizina, diltiazem, piracetam, quinolonas, xantinas, cremes, pomadas e colírios que fazem parte dessa lista de medicamentos genéricos.

\section{Discussão}

Alterações homeostáticas e na farmacocinética e farmacodinâmica de percentual expressivo de medicamentos ocorrem devido ao processo de envelhecimento humano ${ }^{17,18}$. Associadas à ocorrência de alto número de doenças e de fármacos nessa faixa etária, justificam o encontro de duas a três vezes mais interações medicamentosas e reações adversas em idosos do que em adultos jovens ${ }^{19,20}$. O histórico medicamentoso em pacientes idosos deve ser revisto periodicamente na prática clínica. Estima-se que aproximadamente $90,0 \%$ dos idosos façam uso de pelo menos um medicamento de forma regular, com consumo médio de três a cinco 6,7,20-24.

Beers et al., após painel com especialistas em farmacologia e em geriatria, publicaram, em $\left.199\right|^{14}$, o primeiro grupo de critérios para determinar o uso de medicamentos inapropriados em idosos asilados. Produziu-se uma lista de 30 fármacos a serem evitados em asilados, independentemente do diagnóstico, dose e freqüência de sua utilização. Essa lista abrangia psicofármacos, anti-hipertensivos, hipoglicemiantes orais, antiinflamatórios não-hormonais e analgésicos. Revisão desses critérios foi publicada em $19977^{15} \mathrm{com}$ o propósito de incluir idosos em diferentes graus de fragilidade e moradores tanto domiciliarmente como em asilos. Classificou-se os fármacos potencialmente inapropriados em três categorias: I) a serem evitados em idosos em geral; 2) com doses máximas reajustadas pela idade do paciente e 3) a serem evitados em determinadas doenças. Ambas as versões desses critérios foram utilizadas em estudos epidemiológicos ou retrospectivos, sendo incomum o encontro de observações relacionadas com doses medicamentosas e/ou doenças associadas ${ }^{13}$. Revistos por Fick et al., em 2002 ${ }^{16}$, com a finalidade de atualizar fármacos e doenças, essa nova versão reduziu as categorias a duas: I) Medicamentos ou classes deles que deveriam ser evitados em idosos, independentemente do diagnóstico ou da condição clínica, devido ao alto risco de efeitos colaterais e pela existência de outros fármacos mais seguros; 2) Medicamentos ou classes deles que não devem ser usados em determinadas circunstâncias clínicas. Merece menção que as três versões desses critérios não indicam todas as principais causas de prescrição potencialmente inapropriada em idosos como interações medicamentosas. Há também controvérsias sobre a inclusão de alguns desses fármacos, como amitriptilina, largamente utilizada em quadros álgicos em ido$\operatorname{sos}^{13}$. Esses critérios abrangem apenas prescrições inapropriadas, não citando situações como subdosagens medicamentosas e fitoterápicos. Nota-se, ainda, que os critérios de Beers-Fick não apontam para todas as situações que envolvem uso inapropriado de medicamentos em idosos no Brasil. Fármacos de uso comum em nosso país como antitussígenos, cinarizina, diltiazem, piracetam, quinolonas, xantinas, cremes, pomadas e colírios encontrados nessa lista de medicamentos genéricos devem, sob certos critérios clínicos, ser prescritos com cautela nessa faixa etária, dado não relatado em várias de suas bulas nem citado nesses critérios. Cita-se como exemplo a ação farmacológica de certos colírios, potenciais desencadeadores de alterações cardiovasculares e distúrbios psiquiátricos em pacientes idosos ${ }^{25,26}$.

Por outro lado, listas de medicamentos impróprios para idosos, como os critérios de Beers-Fick' ${ }^{16}$, são úteis, de fácil memorização e utilizáveis em vários idiomas 7,23,24,27-28. Deve-se, porém, ter em mente que a idade e o número de fármacos tomados pelo paciente provocam maior risco do uso de medicamentos potencialmente inapropriados $^{23,29}$, exigindo sempre a apreciação de critérios clínicos para melhor seleção de opções terapêuticas. 


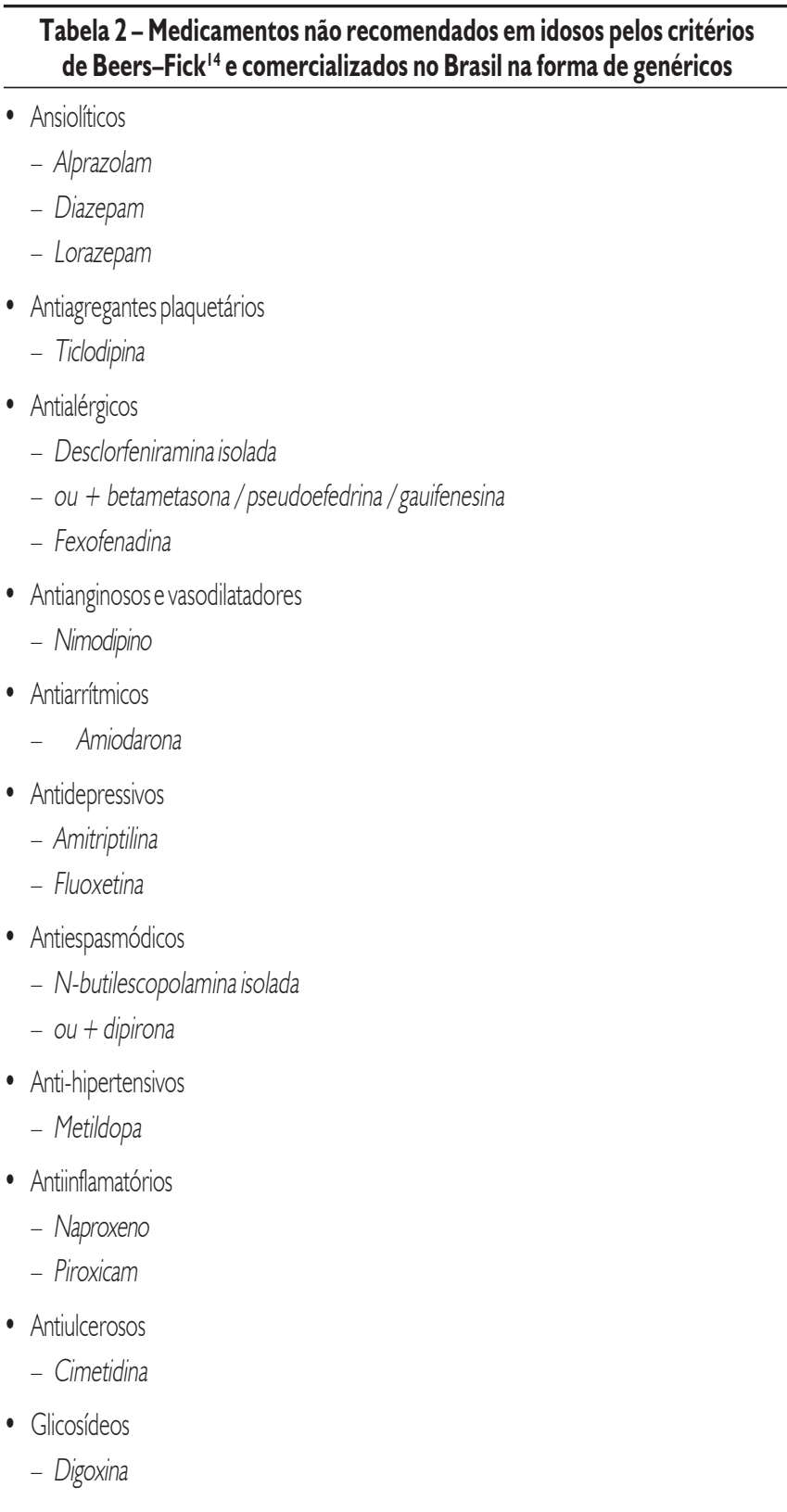

\section{Conclusão}

Critérios de Beers-Fick são úteis para a prevenção do uso de fármacos potencialmente inapropriados em idosos e há medicamentos, incluídos nesses critérios, que são comercializados como genéricos no Brasil.

\section{Agradecimento}

Agradecemos ao Núcleo de Apoio à Publicação da Faculdade de Ciências Médicas da Santa Casa de São Paulo - NAP-SC o suporte técnico-científico à publicação deste manuscrito

\section{Conflito de interesse: não há}

\section{SUMMARY}

BeEr-Fick CRIteria and generic dRUgs in Brazil

BACKGROUND. Determine, according to the Beer-Fick criteria, the prevalence of drugs potentially inappropriate for the elderly available as generic medication in Brazil.

METHODS. Analysis of the list of generic medications issued by "Diário Oficial da União" on July/I2/2004 and of the page of the National Agency for Sanitary Surveillance (ANVISA) - www. anvisa.gov.br, using the BeersFick criteria.

RESULTS. From the list of 299 products 20 (6.7\% of the total) included in the Beers-Fick criteria were analyzed, mainly in the categories of anxiolytics, platelet antiaggregants, antiallergics, anti-angina and vasodilators, antiarrythmics, antidepressants, antispasmodics, antihypertensive's, non steroid antinflammatories, antiulceratives and cardiac glycosides. These criteria do not include drugs such as cough suppressants, cinnarizine, diltiazem, piracetam, quinolones, xanthines, creams, ointments and ophthalmic solutions which are also present in the list of genericmedication.

CONCLUSION. The Beers-Fick criteria may prevent use of drugs potentially inappropriate for the elderly, however, it should be stressed that these criteria are not complete for Brazilian generic medications. [Rev Assoc Med Bras 2008; 54(4): 353-6]

KEY WORDS: Elderly. Drugs. Prescription. latrogeny.

\section{REFERÊNCIAS}

I. Gorzoni ML, Lima CA. Análise dos parâmetros clínicos de idosos internados em enfermaria de clínica médica. Rev Assoc Med Bras. 1995;41:227-32.

2. Straand J, Rokstad KS. Elderly patients in general practice: diagnoses, drugs and inappropriate prescriptions. A report from the More \& Romsdal prescription study. Fam Pract. 1999; 6:380-8.

3. Siqueira AB, Cordeiro RC, Perraccini MR, Ramos LR. Impacto funcional da internação hospitalar de pacientes idosos. Rev Saude Publica. 2004;38:687-94.

4. Montamat SC, Cusack B. Overcoming problems with polypharmacy and drug misuse in the elderly. Clin Geriatr Med. 1992;8: 143-58.

5. Carvalho-Filho ET, Saporetti L, Souza MA, Arantes AC, Vaz MY, Hojaiii $\mathrm{NH}$, et al. latrogenia em pacientes idosos hospitalizados. Rev Saude Publica. 1998;32:36-42.

6. Flaherty JH, Perry HM III, Lynchard GS, Morley JE. Polypharmacy and hospitalization among older home care patients. J Gerontol A Biol Sci Med Sci. 2000;55:M554-9.

7. Klarin I, Wimo A, Fastbom J. The association of inappropriate drug use with hospitalisation and mortality: a population-based study of the very old. Drugs Aging. 2005;22:69-82.

8. Einarson TR. Drug-related hospital admissions Ann Pharmacother. 1993;27:832-40.

9. Lazarou J, Pomeranz BH, Corey PN. Incidence of adverse drug reactions in hospitalized patients: a meta-analysis of prospective studies. JAMA. 1998;279:1200-5.

I0. Doucet J, Chassagne P, Trivalle C, Landrin I, Pauty MD, Kadri N, et al. Drug-drug interactions related to hospital admissions in older adults: a prospective study of I 000 patients. J Am Geriatr Soc. 1996;44:944-8.

I I. Passarelli MC, Jacob-Filho W, Figueras A. Adverse drug reactions in an elderly hospitalised population: inappropriate prescription is a leading cause. Drugs Aging. 2005;22:767-77.

12. Juurlink DN, Mamdani M, Kopp A, Laupacis A, Redelmeier DA. Drug-drug interactions among elderly patients hospitalized for drug toxicity. JAMA. 2003;289: 1652-8 
13. Gallagher P, Barry P, O'Mahony D. Inappropriate prescribing in the elderly. J Clin Pharm Ther. 2007;32: I I 3-21.

14. Beers MH, Ouslander JG, Rollingher I, Reuben DB, Brooks J, Beck JC Explicit criteria for determining inappropriate medication use in nursing home residents. UCLA Division of Geriatric Medicine. Arch Intern Med. |99|; | 51: | 825-32.

15. Beers $\mathrm{MH}$. Explicit criteria for determining potentially inappropriate medication use by the elderly. An update. Arch Intern Med. 1997; I57: I53I-6.

16. Fick DM, Cooper JW, Wade WE, Waller JL, Maclean JR, Beers MH. Updating the Beers criteria for potentially inappropriate medication use in older adults: results of a US consensus panel of experts. Arch Intern Med. 2003; | 63:27| 6-24.

17. Gorzoni ML. Aspectos de farmacologia clínica em pacientes idosos. Gerontologia. 1993; 1:9-12.

18. Oskvig RM. Special problems in the elderly. Chest. 1999; I I 5: I58S-64S

19. Lamy PP. Adverse drugs effects. Clin Geriatr Med. 1990:6:293-307.

20. Mosegui GB, Rozenfeld S, Veras RP, Vianna CM. Avaliação da qualidade do uso de medicamentos em idosos. Rev Saude Publica. 1999;33:437-44.

21. Golden AG, Preston RA, Barnett SD, Llorente M, Hamdan K, Silverman MA. Inappropriate medication prescribing in homebound older adults. J Am Geriatr Soc. 1999;47:948-53.

22. Linjakumpu T, Hartikainen S, Klaukka T, Veijola J, Kivela SL, Isoaho R. Use of medications and polypharmacy are increasing among the elderly. J Clin Epidemiol. 2002;55:809- 17.
23. Chang CM, Liu PY, Yang YH, Yang YC, Wu CF, Lu FH. Potentially inappropriate drug prescribing among first-visit elderly outpatients in Taiwan. Pharmacotherapy. 2004:24:848-55.

24. Ay P, Akici A, Harmanc H. Drug utilization and potentially inappropriate drug use in elderly residents of a community in Istanbul, Turkey. Int J Clin Pharmacol Ther. 2005; 43:195-202.

25. Shiuey Y, Eisenberg MJ. Cardiovascular effects of commonly used ophthalmic medications. Clin Cardiol. 1996; 19:5-8

26. Diamond JP. Systemic adverse effects of topical ophthalmic agents. Implications for older patients. Drugs Aging. 1997; 1 1:352-60.

27. Fialova D, Topinkova E, Gambassi G, Finne-Soveri H, Jonsson PV, Carpenter I, et al. Potentially inappropriate medication use among elderly home care patients in Europe. JAMA. 2005;293: 1348-58.

28. Lechevallier-Michel N, Gautier-Bertrand M, Alperovitch A, Berr C, Belmin J, Legrain S, et al. Frequency and risk factors of potentially inappropriate medication use in a community-dwelling elderly population: results from the 3C Study. Eur J Clin Pharmacol. 2005;60:8 I3-9.

29. Stuck AE, Beers MH, Steiner A, Aronow HU, Rubenstein LZ, Beck JC. Inappropriate medication use in community-residing older persons. Arch Intern Med. 1994; 1 54:2 195-200.

Artigo recebido: 15/10/07

Aceito para publicação: 07//2/07 